between the alloy and overlayer structures. The behavior of the patterns also responds to temperature changes.

\section{Specific Chemical Reactions Induce Reorientation of Nanoscale Liquid Crystals}

Nicholas L. Abbott of the University of Wisconsin-Madison and Rahul R. Shah of the 3M Corp. (formerly of University of Wisconsin) have devised a portable chemical sensor that relies on liquid crystals formed from molecules weakly tethered to a nanotextured surface. As reported in the August 17 issue of Science, the device consists of an ultrathin gold film with nanoscale corrugation. The surface of the gold film is then dotted with protruding chemical receptors that weakly anchor the liquid crystal in a well-defined orientation along the film's surface. When these receptors are exposed to the specific chemical that is the object of detection, however, they bond more strongly with that target chemical than they do with the liquid crystal. The liquid crystal is then displaced into a new orientation that is controlled by the underlying surface texture, changing the sensor's color or brightness. On a surface with car- boxylic acid receptors, for example, exposure to a vapor of the chemical hexylamine caused the liquid crystal to shift from an orientation perpendicular to the gold film's corrugations to an orientation that was parallel with the corrugations. According to the researchers, the "competitive binding" mechanism also allows the sensor to tolerate nontargeted compounds, such as water, which can interfere with detection in other types of sensors. In this case, the nontarget forms an even weaker bond with the receptor than the liquid crystal, and is unable to dislodge it.

\section{Elasticity of Hexagonal Close- Packed Iron at Earth's Inner Core Characterized}

Researchers have been puzzled as to why, based on seismological measurements, elastic waves generated by earthquakes travel through the inner core faster along directions parallel to Earth's polar axis than in other directions. The cause of this difference has not been well understood, partly because the elastic properties of iron at the high pressure and temperature of Earth's center are not known. A research team from the Uni- versity of Michigan, Carnegie Institution of Washington, California Institute of Technology, National Institute of Standards and Technology, and the University of Pennsylvania has used supercomputer simulations to study changes in the crystal structure of iron at high pressure and very high temperatures of 4000-7000 K. As reported in the September 6 issue of Nature, their results support the hypothesis that the directional behavior in seismic wave propagation reflects the alignment of crystals in the inner core. The strong temperature-dependence of the average seismic wave velocity in iron and an almost perfect agreement of such properties with those of the inner core at a temperature of $5700 \mathrm{~K}$ have also led the researchers to infer that this is the temperature in the center of the Earth.

Developing a method that uses a firstprinciples treatment of the electronic structure with an efficient model of the lattice vibrations, the researchers were able to characterize the structure and elasticity of dense hexagonal close-packed (hcp) iron at high temperatures. They calculated the elastic-constant tensor by applying small magnitude finite strains to

\section{Introducing:}
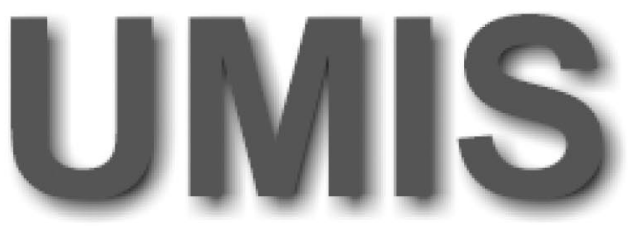

Ultra-Micro Indentation System
All the features you would expect in a nanoindentation test instrument plus the quality and reliability of the original UMIS.

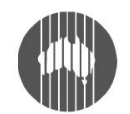

C S IRO

For more information, contact Dr. Tony Fischer-Cripps CSIRO Telecommunications \& Industrial Physics PO Box 218 Lindfield NSW 2070 Australia

Tel: +61294137544

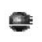

- Cons

- Simulation mode

- Force and depth control

- Independent measurement of indenter load

- Analysis of data from Berkovich, spherical, cube corner and Knoop indenters

- Computerised in-situ video system

- \pm 0.1 um resolution sample positioning

- Integrated WinUMIS 2.0 software combines instrument control and data analysis

e Optional AFM/optical objective
- More features - Higher specifications - Lower price!

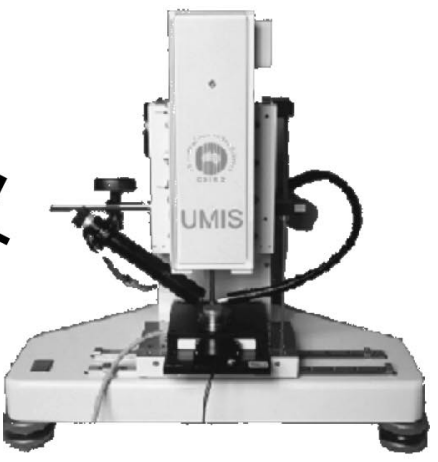

Dual ranges:

Force: $10 \mathrm{mN} \pm 5 \mathrm{nN} 100 \mathrm{mN}$ $\pm 50 \mathrm{nN}$ (up to $1 \mathrm{~N}$ optional)

Depth: $2 \mu \mathrm{m} \pm 0.03 \mathrm{~nm}, 20 \mu \mathrm{m}$ $\pm 0.3 \mathrm{~nm}$

www.tip.csiro.au/umis 